\title{
Multi-indicator design and assessment of sustainable diet plans
}

\author{
Luca Benvenuti $^{1}$
}

\author{
Alberto De Santis ${ }^{1}$
}

April 29, 2021

\author{
Paola Cacchione ${ }^{1}$
}

\begin{abstract}
In this work, a set of diet plans that are nutritionally adequate and healthy, affordable, and environmentally friendly is analyzed. They refer to two-week full-board menus for nursing homes and are designed using a multi-objective optimization model in which different conflicting goals, such as the carbon and water footprints, cost, and nutritional and acceptability requirements, are pursued. As a consequence, the design provides a set of several menus corresponding to different trade-offs between the goals.

Energy and nutrient contents are constrained in suitable ranges suggested by the dietary recommendation of health authorities. The carbon and water footprints and the cost of the diet are considered as goals to be minimized.

More than one hundred menus have been considered to explore all the possible tradeoffs between the three goals. The analysis shows that cheaper menus are more environmentally impacting and that it is possible to make a trade-off between the carbon and water footprints while keeping the cost of the menu unchanged. Moreover, it is possible to quantify the cost increase for a given improvement either of the carbon or water footprint. On the other hand, all the menus are nutritionally adequate and, more importantly, energy and nutrient contents are almost the same despite the large differences in their carbon and water footprints, and cost.

Another interesting result of this study is the analysis of the relationships between the energy and nutrient intakes of the optimal menus and their cost and environmental impacts.
\end{abstract}

Keywords: Food consumption pattern; Environmental sustainability; Economic sustainability.

\section{Introduction}

Sustainability, as stated in 1987 in the Brundtland Report (UN, 1987), consists of "meeting the needs of the present without compromising the ability of future generations to meet their needs." This definition determines a moral responsibility of contemporary society for the

\footnotetext{
${ }^{1}$ Department of Computer, Control, and Management Engineering - Sapienza University of Rome, Via Ariosto 25, 00185 Roma, Italy.
} 
preservation of resources, the environment, and several other ingredients needed for future populations to experience a quality of life good at least as ours.

The natural resource depletion and adverse impacts of environmental degradation due to climate change such as desertification, drought, land degradation, freshwater scarcity, and loss of biodiversity that we are experiencing nowadays show that sustainability is as relevant today as ever. This calls for urgent and significant actions as declared by the United Nations in the 2030 Agenda for Sustainable Development (UN, 2015). One goal of this Agenda is related to achieving food security and improved nutrition as well as promoting sustainable agriculture and sustainable food production systems. There is a close linkage among agriculture, health, the environment, and food consumption and production systems. This led to a more comprehensive definition of sustainable diets, as those "optimizing natural and human resources and that are protective and respectful of biodiversity and ecosystems, culturally acceptable, accessible, economically fair and affordable, nutritionally adequate, safe and healthy" (FAO, 2010).

There are a lot of opportunities that may be viable in the long term in addressing the sustainability of food production and consumption: implement resilient agricultural practices and technologies that increase productivity and production; reduce per capita food waste at the retail and consumer levels and food losses along production and supply chains; shift toward more sustainable food consumption patterns; just to mention a few. In particular, a substantial shift in people eating patterns may help in improving both nutrition quality through betterbalanced diets and the sustainability of the food production systems. As a matter of fact, about one billion people still suffer from hunger, while even more people are overweight or obese (FAO, 2010). Moreover, consistent evidence indicates that a dietary pattern higher in plantbased foods (e.g., vegetables, fruits, legumes, seeds, nuts, whole grains) and lower in animalbased foods (especially red meat), as well as lower in total energy, is both healthier and associated with a lesser impact on the environment (Nelson et al., 2016; USDA, 2015).

In this context, this study aims to explore the relationships between the environmental impact and the cost of diet plans. This is achieved through the diet modeling approach proposed in Benvenuti and De Santis (2020). This approach, given a database of recipes, allows designing realistic menus whilst considering several indicators regarding their health, nutrition adequacy, acceptability, affordability, and environmental impact. It consists of a binary integer linear multi-objective optimization problem in which the values of some indicators are constrained in suitable ranges while others are optimized and considered as goals. It may be considered as a potential model of combined multi-indicator design and assessment of sustainable diet plans.

The approach used in this paper is different from the usual mathematical optimization models that have been developed in the last decade. These models consist of linear programming problems (LP) in which the variables assume continuous values. Basically, they can provide food plans described as the level of consumption of selected food groups (such as fruit and vegetables, dairy, meat, fish, ... ) or food items (potatoes, carrots, beans, eggs, ... ) for person a day or a week (van Dooren, 2018; Gazan et al., 2018).

In general, no realistic menus - that is schedules of recipes - are provided when using LP. Indeed, only Macdiarmid et al. (2012) and Eustachio Colombo et al. (2020) proposed a sample menu to test the practical implementation of the food items and the corresponding 
consumption levels in the optimal food plan. Hence, this step requires the help of an experienced and creative meal planner.

Another key feature of the used method is that it addresses acceptability in a very natural way as opposed to LP that, according to van Dooren (2018), has not provided an ultimate solution to take into consideration acceptability issues.

The design of full-board menus over 14-days for nursing homes is considered. Nutritional adequacy is guaranteed by conforming energy and nutrient intakes to dietary recommendations. Cultural habits and acceptability are instead achieved by limiting in the menu the repetitions of each recipe or recipes in the same food group. The environmental indicators, and in particular carbon and water footprints, as well as the cost of the menu, are considered as goals and optimized to make a trade-off between them.

This case study is an extension of that proposed in Benvenuti et al. (2019) where only two indicators were optimized. The multi-objective optimization problem does not provide a single menu that is the best with regard to all the goals. Rather, it is possible to compute a set of menus for which none of the goals can be decreased in value without increasing some of the others.

More than one hundred menus have been designed to explore all the possible trade-offs between the three goals.

The analysis shows that cheaper menus are more environmentally impacting and that it is possible to make a trade-off between the carbon and water footprints while keeping the cost of the menu unchanged. Moreover, it is possible to quantify the cost increase for a given improvement either of the carbon or water footprint. This provides the management with helpful support to evaluate the costs of more sustainable food services. This shift towards more sustainable menus is supported by the fact that energy and nutrient contents are almost the same for all the menus despite the large differences in their carbon and water footprints and cost. Therefore, the nutritional adequacy of the diet plan is anyway ensured.

Another interesting result of this study is the analysis of the relationships between the energy and nutrient intakes of the optimal menus and their cost and environmental impacts.

\section{Methods and materials}

A menu consists of providing the composition of the meals and their sequence over a given period. To this end, the meals can be composed by selecting dishes from a given set of recipes. This selection must comply with several features related to nutritional adequacy, health, cultural habits, and variability of the menu. Moreover, also the cost of the menu must be considered as well as its environmental impact.

In this study, the design of menus for nursing homes is considered. The food plan is a fullboard menu over 14-days (7 days a week for 2 weeks). A national sample of Italian nursing home menus was collected and 142 different dishes of fixed size (corresponding to one portion) were extracted from them to have a sufficiently large number of recipes to guarantee several choices for breakfast, lunch, and dinner.

To take into account cultural habits and to ensure the attractiveness and variability of the menu, every dish is characterized by its role in the meal and its main ingredient. Hence, for 
example, every breakfast must be composed of some cereals (cornflakes, biscuits, rusks, ... ), a beverage (milk, coffee, tea, juice, ...), and a sweetener (sugar, honey, jam, ... ). On the other hand, lunches and dinners must be composed of a first course, a second course, a side dish, bread, and fruit.

The selected set of recipes contains 47 first courses, 53 second courses, 14 side dishes, 2 possible different kinds of bread, and 9 different fruits. In general, as shown in Figure 1, the first courses include pasta or other carbohydrate sources, the second courses are a source of proteins while the side dishes include vegetables and so provide a small intake of both carbohydrates and proteins. Moreover, the selected dishes are grouped into 18 food groups according to their main ingredient: pasta, rice, soup, red meat, white meat, processed meat, fish, eggs, cheese, potatoes, legumes, zucchini, spinaches, eggplants, mushrooms, artichokes, tomatoes, and tuna fish.

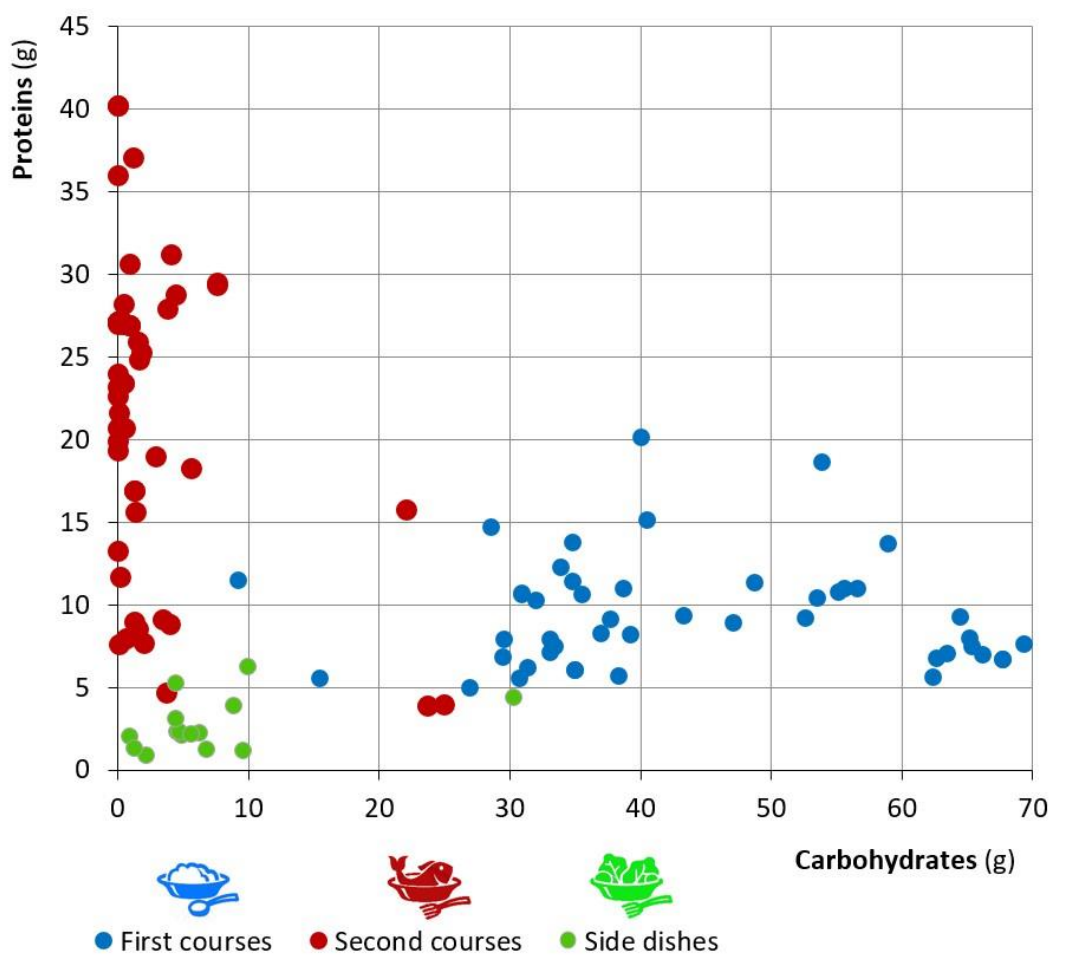

Figure 1: Carbohydrates and proteins of first-course dishes, second-course dishes, and side dishes (one fixed-size portion).

The list of dishes used to design the menu is presented in Table 5 in the Supplementary Material together with the corresponding energy and nutrient contents (proteins, fats, carbohydrates, and sugars), carbon and water footprint, and cost values. These values were computed by combining those of the ingredients of each recipe according to their weights.

Energy and nutrient contents of the ingredients (for $100 \mathrm{~g}$ of food item) were retrieved from the database of the French Agency for Food, Environmental and Occupational Health \& Safety (ANSES, 2020). The data about the environmental impact of ingredients, that is their carbon and 
water footprint (for $100 \mathrm{~g}$ of food item), were retrieved from the Carbon Scope Data LCI database and the CleanMetrics food carbon emission calculator (CleanMetrics, 2020), and the database of the Water Footprint Network (Water Footprint Network, 2020), respectively.

The carbon footprint is expressed as carbon dioxide equivalent and takes into account all the primary greenhouse gases, i.e., carbon dioxide, methane, and nitrous oxide, emitted to produce the ingredients of the dish. The water footprint is the freshwater withdrawals required to produce the ingredients of the dish.

Figure 2 shows the carbon and water footprints of the second-course dishes grouped according to their main ingredient. As expected, red meat dishes, and in particular dishes whose main ingredient is beef meat, are those with the highest environmental impact. In fact, the water footprint of meat from beef cattle is about $15,400 \mathrm{~m}^{3} /$ ton (as a global average) and is much larger than that of meat from pork and chicken which is 6,000 and 4,300 $\mathrm{m}^{3} /$ ton, respectively (Mekonnen and Hoekstra, 2010). Moreover, the production of beef meat generates about $27 \mathrm{~kg}$ of $\mathrm{CO}_{2, e q}$ per $\mathrm{kg}$ which is more than twice the emissions of pork and nearly four times that of chicken (Hamerschlag, 2011). On the other hand, fish dishes have the lowest water footprint and a quite limited GHG emission level.

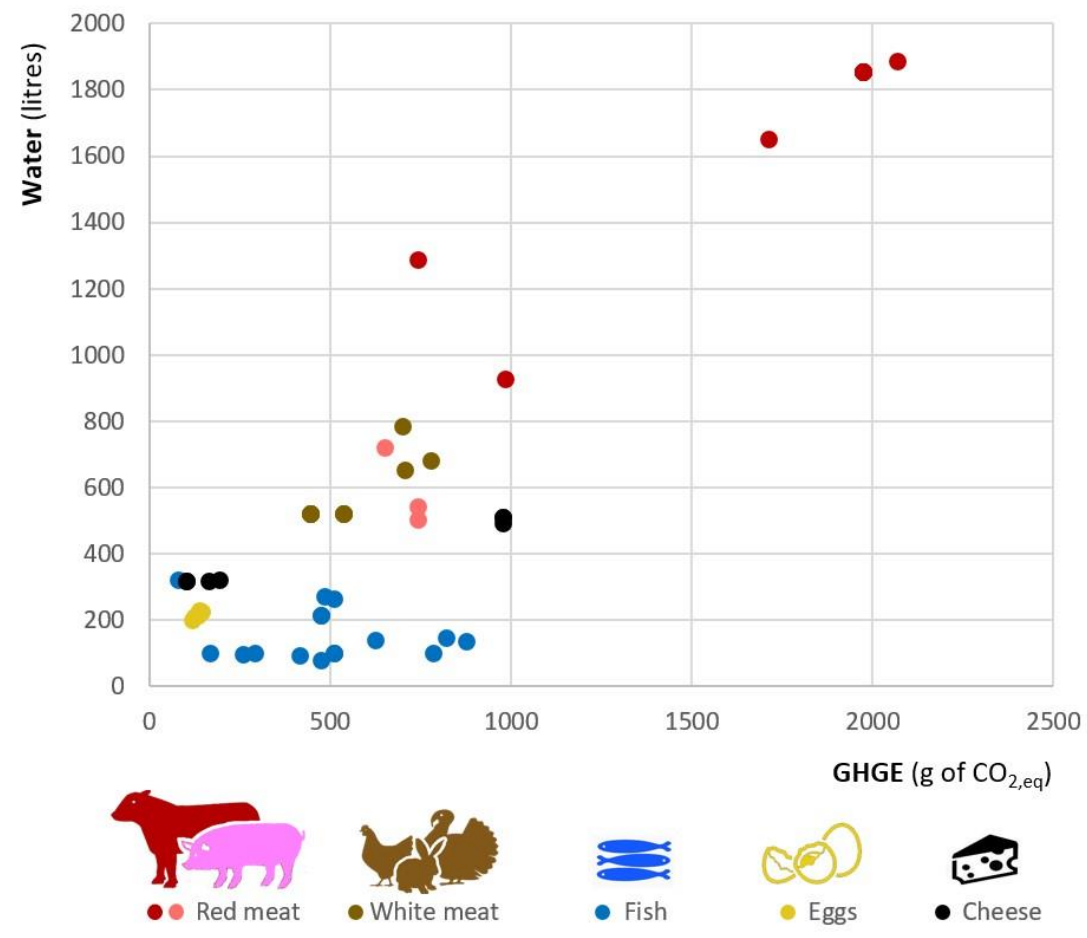

Figure 2: Carbon and water footprints of second-course dishes (one fixed-size portion).

Finally, the cost of every dish is determined by collecting the prices of its ingredients from a sample of local stores considering the mean value price while ignoring prices on specials. 


\section{Nutrition adequacy}

Nutrient adequacy of the menu was calculated according to Italian dietary recommendations (SINU, 2014).

The menu is designed for an energy daily intake of $1,800 \pm 10 \% \mathrm{kcal}$, which corresponds to the reference intake for females $60-74$ years old with a low physical activity level.

The menu consists of breakfast, morning and afternoon snacks, lunch, and dinner. A possible distribution of the daily energy content of at least 10\% from breakfast, about $75 \%$ from lunch and dinner, and the remaining 15\% from snacks (Hermengildo et al., 2016), is considered.

\begin{tabular}{|l|c|c|}
\hline & lower bound & upper bound \\
\hline Energy $(\mathrm{kcal})$ & 1620 & 1980 \\
\hline Carbohydrates $(g)$ & 242 & 296 \\
\hline Proteins $(g)$ & 68 & 103 \\
\hline Fats $(g)$ & 40 & 60 \\
\hline Total sugars $(g)$ & 0 & 93 \\
\hline
\end{tabular}

Table 1: Upper and lower bounds of energy and nutrient daily intakes.

Snacks are chosen to be the same for each day. They include a fruit yogurt pot $(125 \mathrm{~g})$, a cup of tea, and four-five biscuits $(40 \mathrm{~g})$ distributed between morning and afternoon. They provide about $290 \mathrm{kcal}$, which is about $15 \%$ of daily energy content. Consequently, the menu consists of determining only the recipes composing breakfast, lunch, and dinner of each day in the plan. Breakfast is constrained to provide at least $200 \mathrm{kcal}$, which is greater than the recommended amount. Proteins, fats, and carbohydrates provide the most energy according to percentage ranges $10-35 \%, 20-35 \%$, and $45-60 \%$, respectively, as suggested by dietary recommendations (SINU, 2014). Moreover, dietary guidelines require daily sugar intake to be less than $20 \%$ of energy. The resulting upper and lower bounds of energy and nutrient daily intakes are given in Table 1.

\section{Acceptability}

To obtain a varied menu, recipes corresponding to first and second courses cannot be served more than once in the entire menu. On the other hand, since there is a limited number of side dishes, then they can be provided at most once a day but twice a week and three times in the whole menu. The same arguments hold for recipes composing breakfasts. Moreover, dishes in the pasta group cannot be served more than 8 times each week and no more than once a day. The same holds for rice and soup groups. Finally, dishes whose main ingredient is zucchini, spinaches, tuna fish, eggplants, potatoes, mushrooms, artichokes, and tomatoes cannot be served more than once each meal. 


\section{Health security}

Menu health security is addressed according to the recommendations of the World Health Organization (WHO, 2015). Consumption of animal products, especially red and processed meat, is discouraged in favor of the consumption of fish dishes and plant-based foods and, in particular, that of fruits, vegetables, and legumes. Therefore, in the proposed menu, red meat and eggs dishes must be served only once a week. Moreover, dishes of the other food groups can be served according to the repetitions reported in Table 2 .

\subsection{Mathematical modeling and optimization method}

A menu can be designed by selecting dishes from a given set of $N$ recipes of fixed portion size. The design of a menu can therefore be modeled as the assignment of dishes to given places, called slots, in a schedule.

\begin{tabular}{|l|c|c|c|c|}
\hline & \multicolumn{2}{|c|}{ weekly repetition } & \multicolumn{2}{c|}{ total repetition } \\
\hline & $\min$ & $\max$ & $\min$ & $\max$ \\
\hline white meat & 1 & 2 & 3 & 4 \\
\hline processed meat & 0 & 2 & 2 & 3 \\
\hline fish & 2 & 3 & 5 & 6 \\
\hline cheese & 1 & 2 & - & - \\
\hline legumes & 2 & 3 & 5 & 6 \\
\hline
\end{tabular}

Table 2: Upper and lower bounds on the weekly and total serving repetition for some food groups.

The design must comply with health, acceptability, and cultural requirements, as previously described. Moreover, the selection of the dishes is performed trading off the cost and the environmental footprints of the menu. Therefore, to comply with recommendations and selection criteria, some parameters $p$, such as energy and nutrients content (carbohydrates, proteins, fats, and total sugars), cost, and environmental footprints (water and carbon) are associated with each dish.

A slot is identified by a set of three indexes $m, d$, and $w$, denoting the meal $m$ (breakfast, lunch, or dinner) of the day $d$ of the week $w$ in the menu. A binary variable $x_{m, d, w}^{i}$ is associated with every dish and every slot and it assumes value 1 if the dish $i$ is served in the slot $m, d, w$, and 0 otherwise. For example,

$$
x_{\text {lunch }, \text { fri, } 2}^{56}=1
$$

means that dish number 56 is served in the Friday lunch of the second week of the menu. Hence, the design of a menu consists of assigning a value 0 or 1 to all the variables $x_{m, d, w}^{i}$. This must be done in such a way that health, acceptability, and cultural requirements are satisfied. 


\section{Constraints}

The quantity $Q_{d, w}^{p}$ of item $p$ (energy, fats, ..., $C O_{2, e q}$ ) in the day $d$ of the week $w$ is given by

$$
Q_{d, w}^{p}=\sum_{m \in M} \sum_{i=1}^{N} x_{m, d, w}^{i} \times q_{i}^{p}
$$

where $q_{i}^{p}$ is the content of item $p$ in recipe $i$, as reported in Table 5 in the Supplementary Material, and $M$ is the set of meals in a day. Therefore, energy and nutrient intake recommendations determine lower and upper bounds on these quantities, as shown in Table 1.

Moreover, besides energy and nutritional requirements, the schedule must comply with other health recommendations, cultural habits, and eating patterns. These aspects can be taken into account by limiting the number of times (repetition) that each dish can be served in a day, week, or the entire menu, as well as the repetitions of dishes in the same food group $g$ (red meat, rice, ...). The repetition of recipe $i$ in the day $d$ of the week $w$ is given by

$$
R_{d, w}^{i}=\sum_{m \in M} x_{m, d, w}^{i}
$$

so that its weekly and overall repetitions are

$$
R_{w}^{i}=\sum_{d \in D} R_{d, w}^{i}, R^{i}=\sum_{w \in W} R_{w}^{i}
$$

respectively. Similarly, the repetitions of dishes of the food group $g$ in a single meal, day, week, or in the entire menu, can be expressed as follows

$$
R_{m, d, w}^{g}=\sum_{i \in g} x_{m, d, w}^{i}, \quad R_{d, w}^{g}=\sum_{m \in M} R_{m, d, w}^{g}, \quad R_{w}^{g}=\sum_{d \in D} R_{d, w}^{g}, \quad R^{g}=\sum_{w \in W} R_{w}^{g}
$$

Hence, health recommendations and menu acceptability determine lower and upper bounds on these repetitions, as previously described and reported in Table 2 for some food groups.

In conclusion, the nutritional adequacy, health, cultural habits, and variability of the menu can be expressed as lower and upper bounds on the quantities $Q_{d, w}^{p}, R_{d, w}^{i}, R_{w}^{i}, R^{i}, R_{m, d, w}^{g}, R_{d, w}^{g}, R_{w}^{g}, R^{g}$. Note that these constraints turn out to be linear since all the bounded quantities are linear combinations of the variables $x_{m, d, w}^{i}$. 


\section{Selection criteria}

Once all the constraints are determined, all the menus satisfying them are healthy, attractive, and culturally acceptable. These menus will be denoted as "feasible" and differ one to each other either for the served dishes or for the order in which they are served. Moreover, they may have different energy and nutrient contents, different values for the environmental footprints, and different costs. Therefore, it is possible to select a precise menu among all the feasible ones by choosing the "best" one with respect to some goal. For example, it may be possible to choose the menu with minimum cost, or the one with minimum carbon footprint, or that with maximum content of sugar, etc.,.... The goal is usually chosen among the quantities

$$
Q^{p}=\sum_{w \in W} \sum_{d=D} Q_{d, w}^{p}
$$

representing the amount of item $p$ in the entire menu. As above, note that $Q^{p}$ turns out to be a linear combination of the variables $x_{m, d, w}^{i}$. From a mathematical point of view, this kind of selection is then a binary integer linear programming (BLP) problem, which is an optimization problem over binary variables with linear constraints and a linear objective function. The problem is usually denoted as

$$
x^{\star}: f\left(x^{\star}\right)=\min _{x \in F} f(x)
$$

where:

- $x$ denotes a possible menu, that is a set of values of the variables $x_{m, d, w}^{i}$ assigning dishes to all available slots of the menu;

- $F$ represents the set of all the feasible menus;

- $f(x)$ is the so-called objective function, and it represents the goal of the selection criterion, given by (1), which is a linear function of $x$;

- $x^{\star}$ is the "best feasible menu", i.e., the feasible menu that minimizes the goal $f(x)$.

Considering more than one goal gives rise to a multi-objective optimization problem. In this case, the problem is usually denoted as

$$
\min _{x \in F}\left(f_{1}(x), f_{2}(x), \cdots, f_{k}(x)\right)
$$

where, in general, the linear objective functions $f_{i}(x)$ are conflicting, that is no single "best feasible menu" $x^{\star}$ exists that simultaneously optimizes each objective. In this case, a significant set of menus is given by the so-called Pareto optimal solutions. This set consists of all the menus for which none of the objective functions can be improved in value without degrading some of the other goals. It corresponds to a set of compromised trade-off solutions from which the best solution for a particular problem can be chosen rather than considering the whole range of 
feasible menus. The interested reader is referred to Benvenuti and De Santis (2020) for a more detailed description of the model and a complete characterization of the Pareto optimal set.

\section{Results and discussion}

Three indicators were considered as goals in the design of the menu: the cost of the menu and its environmental impact expressed in terms of carbon and water footprints. The Pareto optimal solutions were computed using the $\varepsilon$-constraint method. It consists of minimizing one objective when applying upper bounds to all the others. Hence, the Pareto optimal set is obtained by solving a sequence of scalar optimization problems suitably varying these upper bounds (Miettinen, 1999; Hwang and Masud, 1979).

The optimization was carried out by using the online version of CPLEX for AMPL on the NEOS server and made it possible to compute 119 different menus that are Pareto optimal solutions. These solutions are possibly a subset of the complete discrete Pareto set. There are more efficient methods able to compute the Pareto front in recent literature, see for example Boland et al. (2017).

The energy and nutrient contents as well as the cost and the footprints corresponding to the optimal menus are presented in Table 6 in the Appendix. The cost is the total cost of the ingredients needed to serve the whole menu for one person. Similarly, the footprints are the freshwater used $\left(\mathrm{m}^{3}\right)$ and the $\mathrm{CO}_{2, e q}$ emitted $(\mathrm{kg})$ to serve the whole menu for one person.

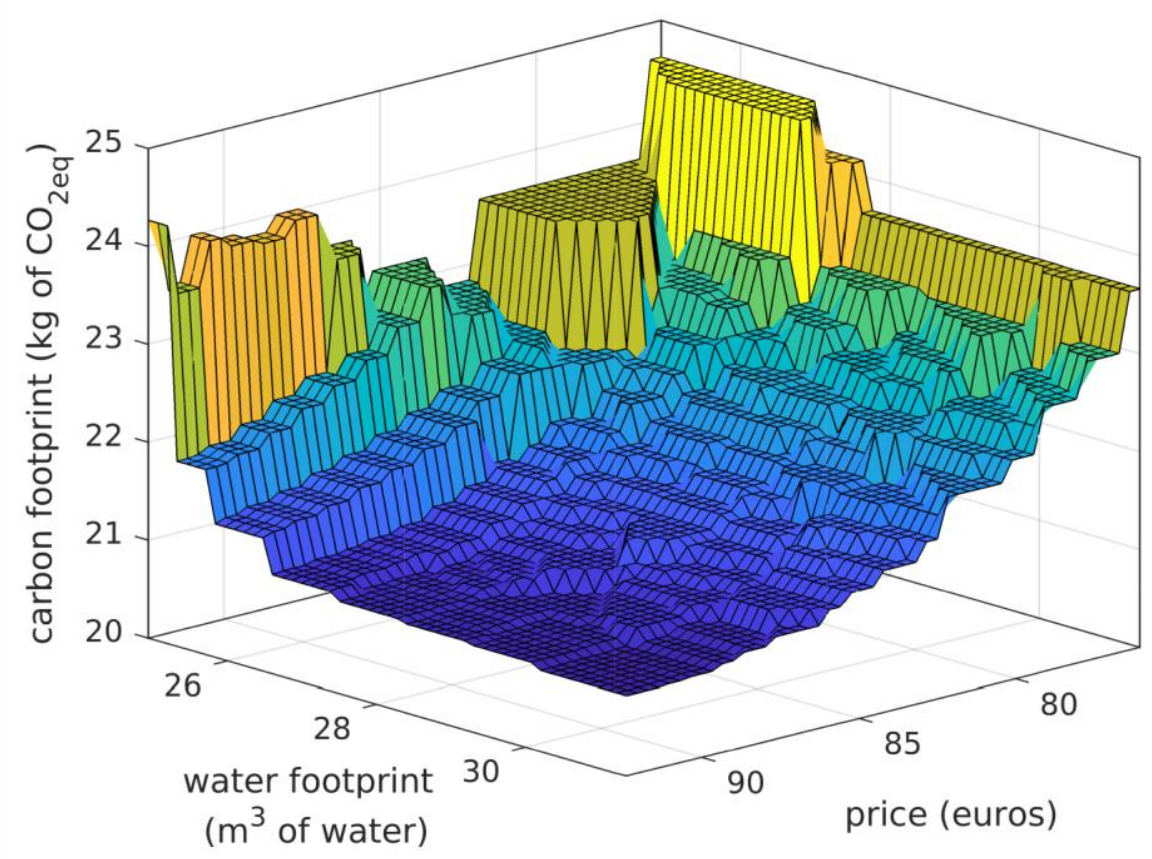

Figure 3: Pareto surface 
Figure 3 shows the Pareto optimal set in the objective function space, that is, the space with one coordinate axis for each goal. The Pareto optimal set, which is usually called the trade-off surface, gives full information on objective values and shows how improving one objective is related to deteriorating the other ones while moving along the trade-off surface. In more detail, the figure shows that cheaper menus are more environmentally impacting and that it is possible to make a trade-off between carbon and water footprints while keeping the cost of the menu unchanged ${ }^{1}$. This can be seen more clearly in Figure 4 where the Pareto surface has been sliced to yield two-dimensional trade-off curves corresponding to menus with the same price (isocost lines).

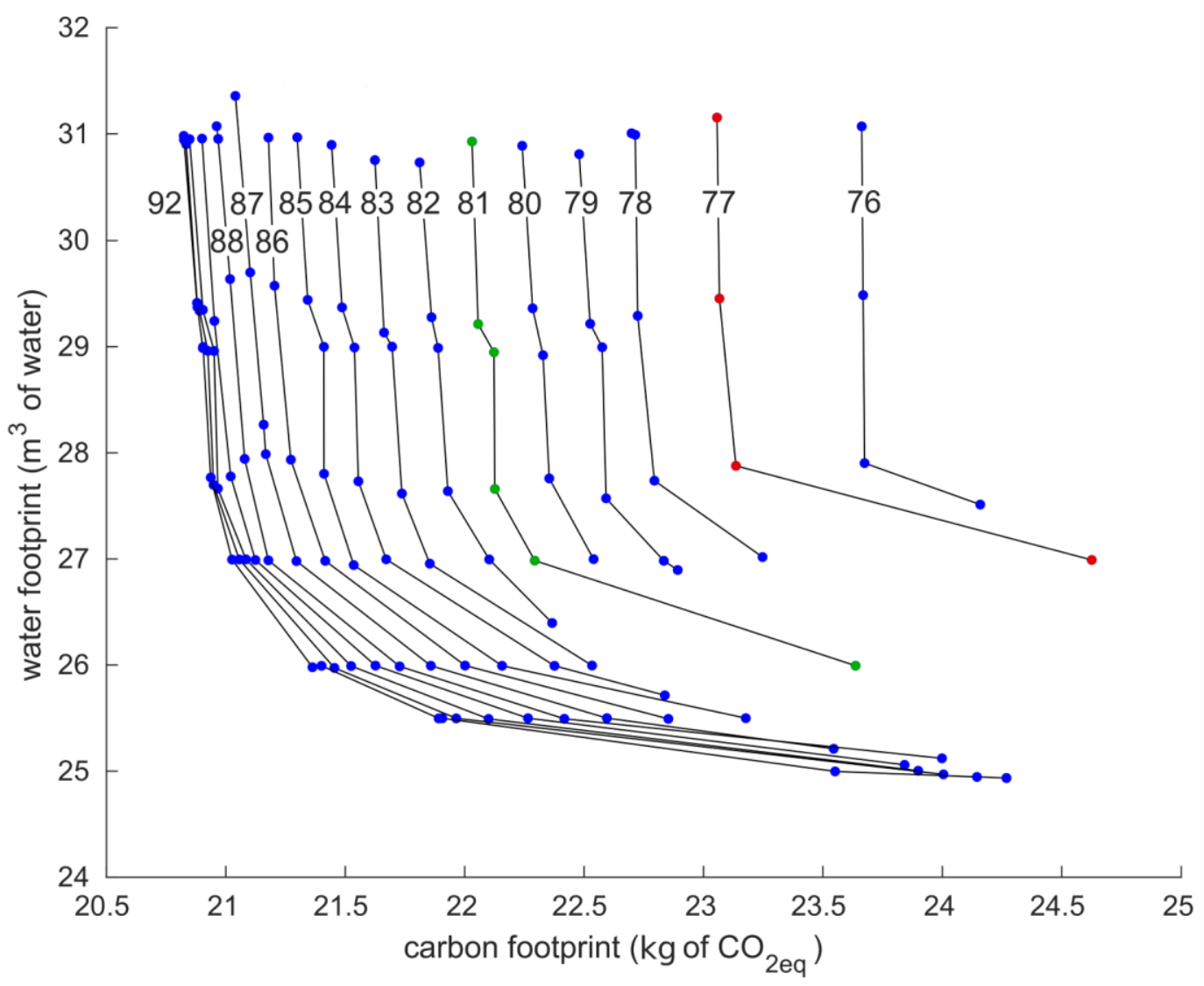

Figure 4: Price isolines

\footnotetext{
${ }^{1}$ This result may appear counter-intuitive since expensive food may be highly impacting, as for example beef meat. Nevertheless, this result concerns the whole menu and not the single food item. Indeed, all the considered menus must include red meat exactly once a week and hence they share the same economic and environmental impact due to this particular food. It is then evident that the difference in cost and environmental impact depends on the other recipes of the menu. In general, menus with a small difference in price are substantially the same, that is only a few recipes are substituted with others and the number of common recipes decreases as the difference in price increases. The interested reader is referred to Benvenuti et al. (2019) where this issue is deepened in the case of the relation between cost and carbon footprint (see Table 2 in Benvenuti et al. (2019)).
} 
This kind of visualization gives a clear picture of trade-offs between the goals and helps quantify the improvement and the corresponding deteriorations of the objective functions when moving from one solution to another one.

As an example, consider the menus whose cost is approximately $77 €$ (see the red points in Figure 4). The menu with minimum carbon footprint at this price, requires about $23 \mathrm{~kg}$ of $\mathrm{CO}_{2, e q}$ while the menu with minimum water footprint at the same price requires about $27 \mathrm{~m}^{3}$ of freshwater (this can be checked also considering menus 5-8 in Table 6 in the Supplementary Material). Increasing the price to $81 €$ (see the green points in Figure 4), makes it possible to reduce the minimum achievable footprints to $22 \mathrm{~kg}$ of $\mathrm{CO}_{2, e q}$ and $26 \mathrm{~m}^{3}$, respectively (this can be checked also considering menus 25-30 in Table 6). Obviously, at a constant price, the menu with the minimum of one environmental footprint requires the maximum of the other one. The figure allows then, once the price has been set, to make a trade-off between the footprints choosing different solutions over the same isocost line.

The fact that isocost lines corresponding to high prices are closer together than those at low prices indicates that when equal price increases are considered, footprint reductions are greater when price increments are made starting from low initial costs.

For example, as shown in Figure 5, a $2 €$ increment of price on the menu that costs $76 €$ and has a water footprint of about $28 \mathrm{~m}^{3}$, produces a reduction of about $3.7 \%$ on the carbon footprint while keeping the water footprint unchanged (see the red points in the figure corresponding to menus 3 and 12 in Table 6). The same price increment on the menu that costs $86 €$ and has the same water footprint, produces a reduction of about $0.9 \%$, instead (see the green points in the figure corresponding to menus 59 and 74 in Table 6).

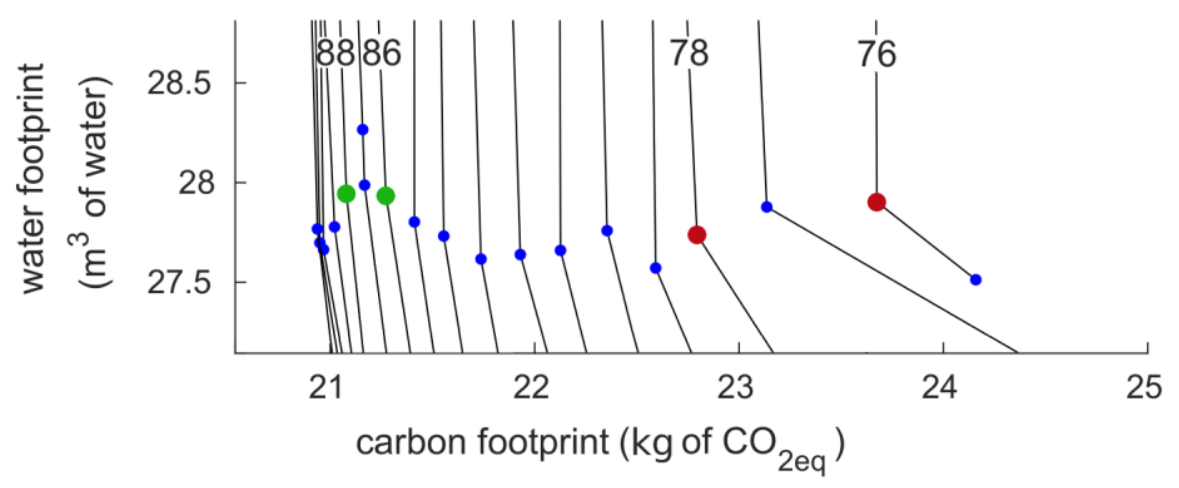

Figure 5: Zoomed part of Figure 4

This is an important result since the usual choice of nursing home management is that of reducing costs while ensuring residents receiving a varied and healthy diet that meets their nutritional needs. Hence, it is just starting from this situation that it is possible to obtain the best environmental impact reduction at the same cost.

Similar figures can be drawn when considering menus with the same carbon or water footprint, but they seem to be less useful than that at a constant price.

Figure 6 evaluates the nutritional adequacy of the Pareto optimal solutions. In this figure, 


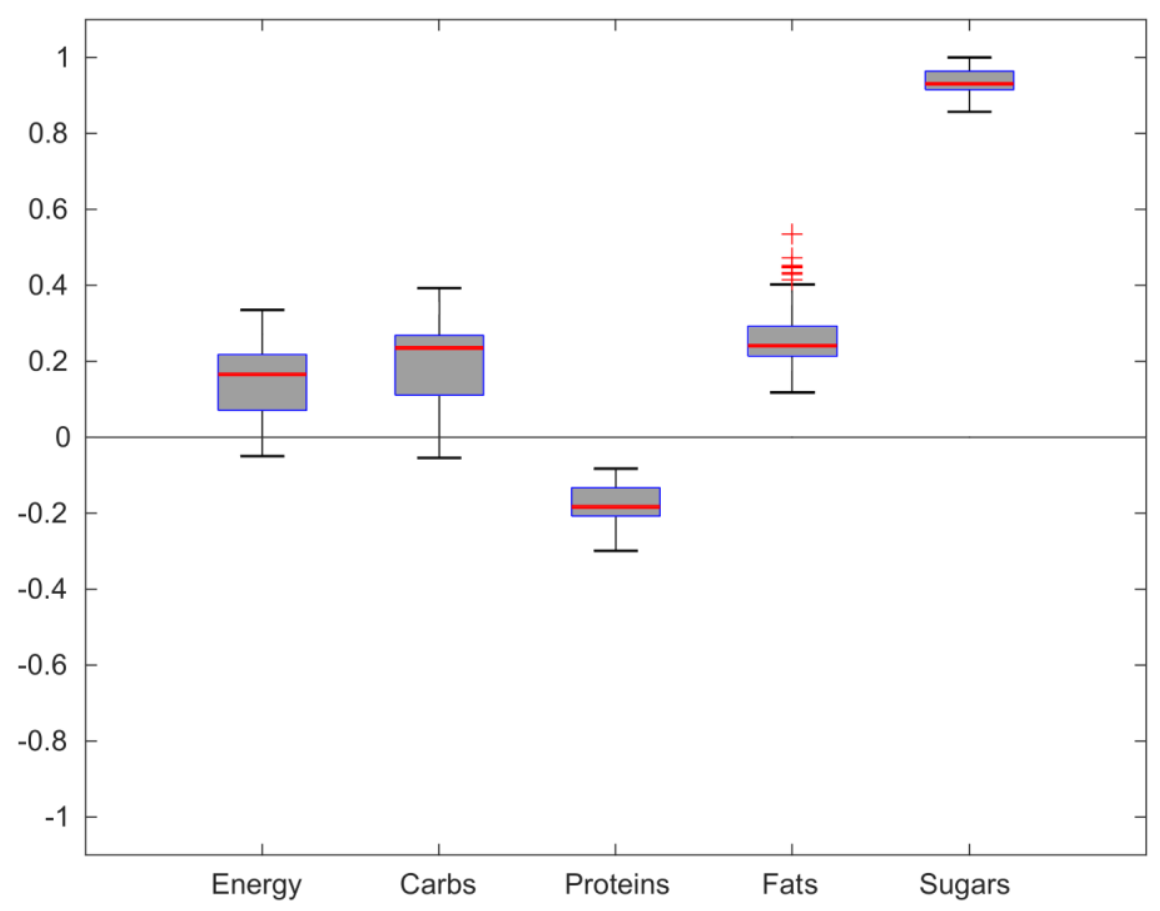

Figure 6: Box-plots of energy and nutritional daily mean contents of the optimal menus. The values are scaled so that -1 and 1 correspond to the daily lower and upper bounds given in Table 1, respectively.

the box plots of energy and nutritional values of the optimal menus are depicted and compared with the allowable maximum and minimum values. In more detail, the values 1 and -1 on the vertical axis of the figure correspond to the daily upper and lower bounds given in Table 1, respectively.

The figure makes clear that the values of the Pareto optimal menus are mainly distributed in a very small range as compared to the reference one. Moreover, apart from sugars, they are very close to the average reference value since the median value differs at most about $\pm 20 \%$ from it. Sugars, that are bounded only from above, are instead very close to the maximum allowable value.

From the above discussion, it follows that the optimal menus are very similar in terms of their nutritional adequacy. This result is quite surprising since it holds notwithstanding the large variations of environmental and socio-economic indicators. Moreover, it implies that the trade-off between the goals can be done without affecting too much the nutritional value of the menus.

Another interesting issue is that of understanding which kind of relationship there is between the nutritional values of the optimal menus and their cost and environmental impact, if any. To this end, the Kendall rank coefficient has been computed for any pair of sets of values 


\begin{tabular}{|c|c|c|c|c|c|c|c|c|}
\hline & Energy & Carbs & Proteins & Fats & Sugars & Carbon FP & Water FP & Cost \\
\hline Energy & 1 & 0.62 & 0.59 & 0.13 & 0.36 & -0.20 & 0.42 & -0.02 \\
\hline Carbs & & 1 & 0.37 & -0.11 & 0.33 & -0.05 & 0.42 & -0.17 \\
\hline Proteins & & & 1 & -0.05 & 0.39 & -0.48 & 0.40 & 0.21 \\
\hline Fats & & & & 1 & -0.07 & 0.26 & -0.01 & -0.20 \\
\hline Sugars & & & & & 1 & -0.24 & 0.31 & 0.10 \\
\hline Carbon FP & & & & & & 1 & -0.32 & -0.48 \\
\hline Water FP & & & & & & & 1 & -0.21 \\
\hline Cost & & & & & & & & 1 \\
\hline
\end{tabular}

Table 3: Kendall's $\tau$ coefficients.

\begin{tabular}{|l|cccccccc|}
\hline & Energy & Carbs & Proteins & Fats & Sugars & Carbon FP & Water FP & Cost \\
\hline Energy & 1 & 0 & 0 & 0.04 & 0 & 0 & 0 & 0.74 \\
Carbs & & 1 & 0 & 0.08 & 0 & 0.39 & 0 & 0.01 \\
Proteins & & & 1 & 0.44 & 0 & 0 & 0 & 0 \\
Fats & & & & 1 & 0.23 & 0 & 0.83 & 0 \\
Sugars & & & & & 1 & 0 & 0 & 0.10 \\
Carbon FP & & & & & & 1 & 0 & 0 \\
Water FP & & & & & & & 1 & 0 \\
Cost & & & & & & & & 1 \\
\hline
\end{tabular}

Table 4: $p$-values.

to establish whether they may be regarded as statistically dependent. Such coefficients are reported in Table 3.

The correlation coefficients measure the strength of association between the values of the two sets and the direction of the relationship. The value of the correlation coefficient varies between +1 and -1 where a value of \pm 1 indicates a perfect degree of association and the sign indicates its direction. The more the correlation coefficient value is close to 0 , the more the relationship between the two sets of values has to be considered weak. The statistical significance of the independence hypothesis (the null hypothesis of no association) is evaluated using the $p$-values, as reported in Table 4.

The pairs energy-carbohydrates and energy-proteins have Kendall rank coefficients equal to 0.62 and 0.60 , respectively, and a $p$-value very close to zero. This suggests a strong relationship between the items in each pair.

The scatter plots of these two pairs are depicted in Figure 7 together with the corresponding regression lines to test if the associations are of linear type. The relationships can then be assumed linear by noting that the resulting coefficients of determination are equal to 0.75 and 
0.62 , respectively. This result is due somewhat to the fact that proteins and carbohydrates are constrained to provide the most energy according to given percentage ranges. Nevertheless, it doesn't hold necessarily true, as can be seen by checking the pair energy-fats for which a similar constraint exists. The values in the tables show that there is an association between the two items ( $p$-value equal to 0.04 ) but the relationship is not linear since the Kendall rank coefficient is equal to 0.13 .
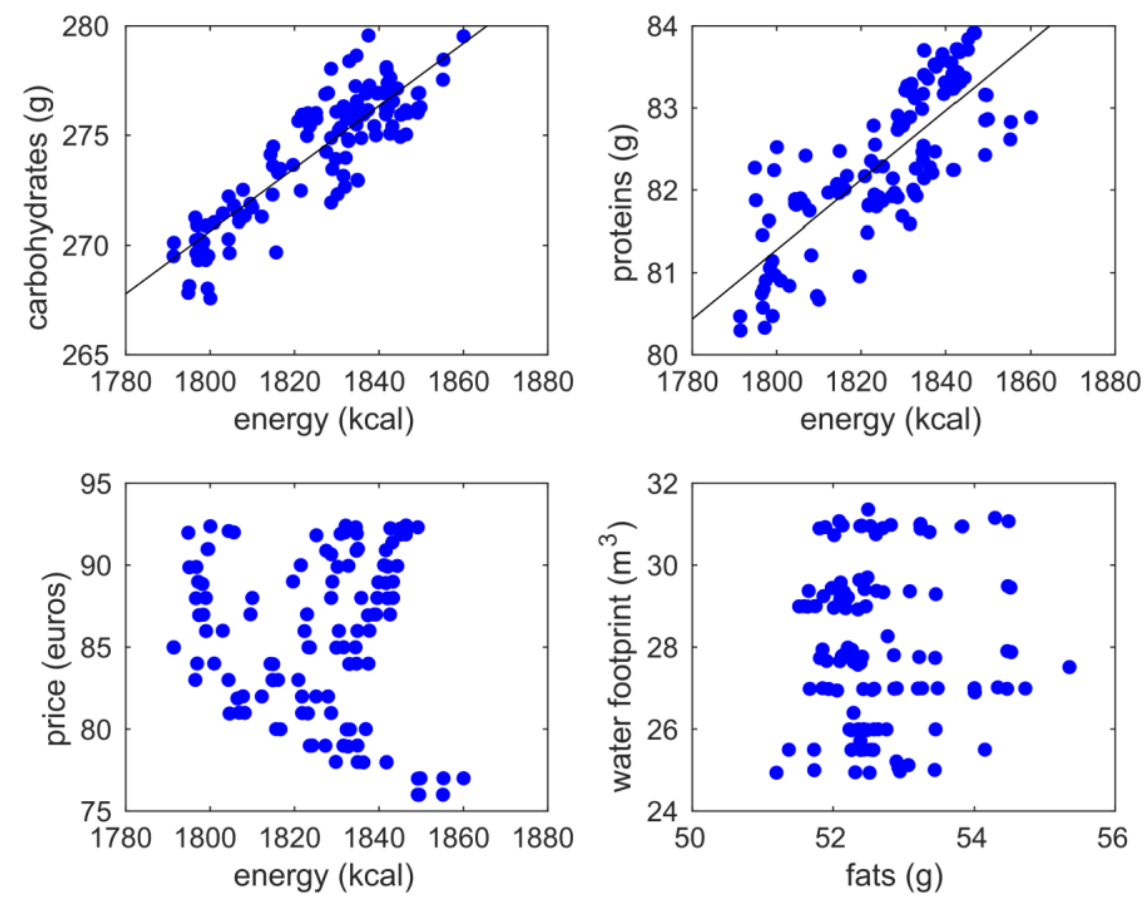

Figure 7: Scatter plots of some pairs of nutritional and environmental indicators.

On the other hand, the Kendall rank coefficients associated with the pairs proteins-fats, carbohydrates-carbon footprint, fats-sugars, fats-water footprint, and energy-cost are close to zero (their modulus are less or equal to 0.07 ) while the corresponding $p$-values are well greater than $5 \%$. Hence, there is no relationship between the items of each of such pairs. This is made evident in Figure 7 where the scatter plots of two of them are depicted.

Finally, note that the $p$-values of the pairs water footprint-carbon footprint, carbon footprint-cost, and cost-water footprint denote association between the indicators. However, the corresponding Kendall rank coefficients are $-0.32,-0.48$, and -0.21 , respectively, thus denoting a nonlinear relationship. Moreover, the negativity of the coefficients implies that an increment of one indicator corresponds to a reduction of the other. This is exactly what the Pareto surface approximation of Figure 3 shows: a hyperbolic-like relationship between them.

\section{Conclusions}

Sustainable diets contribute to food and nutrition security and to a healthy life for present and future generations. They are therefore characterized by low environmental impacts and should 
be culturally acceptable, accessible, economically fair, and affordable. As a consequence, a food plan design has to consider different indicators usually conflicting with each other.

The approach used in this paper allows dealing with several indicators regarding health, nutrition adequacy, acceptability, affordability, and environmental impacts of menus. It consists of a binary integer linear multi-objective optimization problem in which the values of some indicators are constrained in suitable ranges while others are optimized. Nutritional adequacy is guaranteed by limiting energy and nutrient intakes according to country-specific dietary recommendations.

On the other hand, cultural habits are satisfied by considering the country-specific structure of the meals and an appropriate set of dishes to compose the menu. Acceptability is achieved by limiting in the menu the repetitions of each recipe or those of recipes in the same food group. The environmental indicators, and in particular carbon and water footprints, as well as the cost of the menu, are considered as goals and optimized to make a trade-off between them. Indeed, there is not a single menu optimizing all the goals but it is possible to determine a set of menus for which none of the goals can be decreased in value without increasing some of the others.

This approach has been applied to the design and assessment of a two-week full-board menu for nursing homes.

The results show that it is possible to quantify the variations of the goals when moving from one optimal menu to another. It is shown that cheaper menus are more environmentally impacting and that it is possible to make a trade-off between carbon and water footprints while keeping the cost of the menu unchanged.

Moreover, the improvement of environmental impact indicators can be assessed for a given cost increment. It is shown that footprint reductions are greater when price increments are made starting from low initial costs. This is an important result since the usual choice of nursing home management is that of reducing costs while ensuring residents receiving a varied and healthy diet that meets their nutritional needs. Hence, it is just starting from this situation that it is possible to obtain the best environmental impact reduction at the same cost.

Furthermore, it is shown that the optimal menus are very similar in terms of their nutritional adequacy. This implies that the trade-off between the goals can be done without affecting too much the nutritional value of the menus.

The model is fully scalable so that a larger set of dishes can be considered as well as other indicators used. The menu characteristics can be improved by considering either a larger database or specific dishes developed to have low cost or low environmental impacts. Moreover, more nutritional indicators can be used to assess the adequacy of the diet plan (Ribal et al., 2016), or further environmental indicators can be included in the goals. For example, the land use was used in (Ward et al., 2014), but a more complete environmental profile can be considered using, when available, the values of the typical indicators of an LCA or EPD for each food product included in the recipes. Furthermore, smaller ranges for the nutritional indicators can be adopted by allowing several serving sizes (i.e., half or full portion) of each dish.

The model can also comply with different meal structures depending on the eating habits. For example, in Ribal et al. (2016), the lunch is composed of a starter, a main dish, and a dessert. Hence, dishes can be associated with these three different categories where main dishes usually contain a meat or fish recipe and some vegetables. Therefore, energy and nutrient contents, as 
well as price and environmental footprints, are associated, in this case, with the whole dish and not with every single recipe.

Finally, the proposed procedure can be easily applied to some other food service areas such as company service canteens, chain restaurants, or other individual establishments. In these cases, it could be worth considering other acceptability features related to the sensorial perspective. However, introducing these aspects is a complicated task since characteristics such as food aroma, appearance, taste, and texture are typically subjective and hard to quantify. On the other hand, simpler features such as food and wine pairing could be easily introduced in the proposed model.

\section{Funding}

This research was developed within the project SYSTEMIC (An integrated approach to the challenge of sustainable food systems: adaptive and mitigatory strategies to address climate change and malnutrition) that has received funding from national research funding parties in Belgium (FWO), France (INRA), Germany (BLE), Italy (MIPAAF), Latvia (IZM), Norway (RCN), Portugal (FCT), and Spain (AEI) in a joint action of JPI HDHL, JPI-OCEANS, and FACCEJPI launched in 2019 under the ERA-NET ERA-HDHL (n 696295) of the Knowledge Hub on Food and Nutrition Security (KH FNS).

\section{References}

UN (United Nations) 1987, Report of the World Commission on Environment and Development: Our Common Future.

UN (United Nations) 2015, Transforming our world: the 2030 Agenda for Sustainable Development, https://sustainabledevelopment.un.org/post2015/transformingourworld

FAO (Food and Agriculture Organization) 2010, Sustainable diets and biodiversity: directions and solutions for policy, research and action. Proceedings of the International Scientific Symposium Biodiversity and sustainable diets united against hunger, FAO Headquarters, Rome.

Nelson, M. E., Hamm, M. W., Hu, F. B., Abrams, S. A., Griffin, T. S., Alignment of Healthy Dietary Patterns and Environmental Sustainability: A Systematic Review. Advances in Nutrition, 7(6): 1005-1025, 2016.

USDA (US Department of Agriculture and US Department of Health and Human Services) 2015, Dietary Guidelines Advisory Committee. Scientific Report of 2015. Washington (DC).

van Dooren, C., A Review of the Use of Linear Programming to Optimize Diets, Nutritiously, Economically and Environmentally. Frontiers in Nutrition, 5:48, 2018. 
Gazan, R., and Brouzes, C M. C., Vieux, F., Maillot, M., Lluch, A., Darmon, N., Mathematical Optimization to Explore Tomorrow's Sustainable Diets: A Narrative Review. Advances in Nutrition, 9(5): 602-616, 2018.

Macdiarmid, J.I., Kyle, J., Horgan, G.W., Loe, J., Fyfe, C., Johnstone, A., McNeill, G., Sustainable diets for the future: can we contribute to reducing greenhouse gas emissions by eating a healthy diet? The American Journal of Clinical Nutrition, 96 (3), 632e639, 2012.

Eustachio Colombo, P., Patterson, E., Lindroos, A.K., Parlesak, A., Schäfer Elinder, L., Sustainable and acceptable school meals through optimization analysis: an intervention study. Nutrition Journal, 19, 61, 2020.

Benvenuti, L., De Santis, A., Di Sero, A., Franco, N., Concurrent economic and environmental impacts of food consumption: are low emissions diets affordable?, Journal of Cleaner Production, 236, Article 117645, 2019.

ANSES (Agence nationale de sécurité sanitaire de l'alimentation, de l'environnement et du travail) 2020, Table de composition nutritionelle des aliments, https://ciqual.anses.fr

CleanMetrics 2020, Food carbon emissions calculator, CleanMetrics, http://www.foodemissions.com/foodemissions/Calculator

The Water Footprint Network 2020, https://waterfootprint.org

Mekonnen, M. M., Hoekstra, A. Y., The green, blue and grey water footprint of farm animals and animal products. Technical Report 48, Unesco-IHE Institute for Water Education, 2010.

Hamerschlag, K., A meat eaters' guide to climate change + health: what you eat matters, https://www.ewg.org/meateatersguide

SINU (Societa` Italiana di Nutrizione Umana) 2014, Livelli di assunzione di riferimento di nutrienti ed energia per la popolazione Italiana (LARN) IV Revisione (in Italian).

Hermengildo, Y., López-García, E., García-Esquinas, E., Pérez-Tasigchana, R., RodríguezArtalejo, F., Guallar-Castillón, P., Distribution of energy intake throughout the day and weight gain: A population-based cohort study in Spain. British Journal of Nutrition, 115(11), 2003-2010, 2016.

WHO (World Health Organization) 2015, Healthy diet, Fact sheet N. 394, Updated January 2015.

Benvenuti, L., De Santis, A., Making a sustainable diet acceptable: an emerging programming model with applications to schools and nursing homes menus. Frontiers in Nutrition, 7, Article 562833, 2020.

Miettinen, K., Nonlinear Multiobjective Optimization. Springer, 1999.

Hwang, C. L., Masud, A. S. M., Multiple objective decision making, methods and applications: a state-of-the-art survey. Springer-Verlag, 1979. 
Boland, N., Charkhgard, H., Savelsbergh, M., The quadrant shrinking method: A simple and efficient algorithm for solving tri-objective integer programs. European Journal of Operational Research, 260(3): 873-885, 2017.

Ribal, J., Fenollosa, M. L., Garcia-Segovia, P., Clemente, G., Escobar, N., Sanjuan, N., Designing healthy, climate friendly and affordable school lunches. The International Journal of Life Cycle Assessment, 21(5):631-645, 2016.

Ward, J. D., Ward, P. W., Mantzioris, E., Saint, C., Optimising diet decisions and urban agriculture using linear programming. Food Security, 6:701-718, 2014. 